\title{
Spectral Transmission Line Analysis of a Composite Right/Left-Handed Uniaxially Anisotropic Meta-substrate
}

\author{
Attieh Shahvarpour \\ Poly-Grames Research Center, \\ École Polytechnique de Montréal \\ Montréal, QC, H3T 1J4, Canada \\ Email: attieh.shahvarpour@polymtl.ca
}

\author{
Alejandro Alvarez Melcon \\ Technical University of Cartagena, \\ Campus Muralla del Mar-Antiguones, \\ 30202 Cartagena (Murcia), Spain \\ Email: alejandro.alvarez@upct.es
}

\author{
Christophe Caloz \\ Poly-Grames Research Center, \\ École Polytechnique de Montréal \\ Montréal, QC, H3T 1J4, Canada \\ Email: christophe.caloz@polymtl.ca
}

\begin{abstract}
The TM spectral transmission line model of a grounded meta-substrate with uniaxially anisotropic properties, corresponding to a composite right/left-handed sub-wavelength mushroom-type structure, is computed. The dispersion relation is found by the transverse resonance technique. It is analyzed and compared with the particular case of an isotropic grounded slab. The study is limited to the left-handed region of the metasubstrate, which corresponds to the frequency band where the permittivity and permeability are negative and most dispersive. The work considers only TM modes, for which the effects of dispersion and anisotropy are most significant. The leaky-modes in the anisotropic left-handed meta-substrate are shown to exhibit a leakage factor which may be conveniently designed to very low values for super highly directive leaky-wave antennas.
\end{abstract}

\section{INTRODUCTION}

Meta-substrates are artificial dielectric structures with unusual properties [1]-[3]. In particular, they have been proposed as "real material" alternatives to standard substrates with metamaterial patterned strips printed on them [4], since they produce similar effects with uniform strips from their intrinsic structure. Specifically, a composite right/left-handed metasubstrate with uniform microstrip line exhibiting fully-space scanning leak-wave capability was demonstrated in [5]. This meta-substrate has a mushroom-type structure [6], [4] with conducting wires (metalized vias) perpendicular to the airdielectric interface and conducting loops (formed by vias and patches), leading to uniaxially anisotropic permittivity and permeability along the axes of the wires and the plane of the loops, respectively [5]. However, no rigorous method has been reported for the analysis of such a structure.

In this work, a spectral domain transmission line approach [7] is used for a rigorous analysis of a meta-substrate with the same anisotropy as in [5]. The TM dispersion relation of the meta-substrate is found by the transverse resonance technique [8]. Only TM modes, for which the effects of dispersion and anisotropy are most significant, are considered. The dispersion relation is analyzed in the left-handed region of the structure, which corresponds to the frequency band where the permittivity and permeability are negative and most dispersive. The dispersion of the anisotropic meta-substrate is compared with those of an isotropic left-handed grounded slab [9].

\section{II. $\mathrm{TM}_{z}$ SPECTRAL TRANSMISSION LINE EQUATIONS OF an Unbounded UniaXially ANISOTROPIC Medium}

Assuming the time dependence $\exp (+j \omega t)$, the spectral domain transmission line equations have the form [7]

$$
\begin{aligned}
& \frac{d \tilde{I}}{d z}=-j Y k_{z} \tilde{V}, \\
& \frac{d \tilde{V}}{d z}=-j Z k_{z} \tilde{I},
\end{aligned}
$$

where $\tilde{V}, \tilde{I}, Z, Y$ and $k_{z}$ are the spectral-domain voltage and current, impedance, admittance and the wave number along the $z$ axis of the transmission line, respectively. The characteristic impedance $Z_{c}=\sqrt{Z / Y}$ and the propagation constant $\beta=\sqrt{Z Y} k_{z}$ of the transmission line can be calculated from the solutions of the transmission line equations $\tilde{V}=\tilde{V}^{+} \exp \left(-j \sqrt{Z Y} k_{z} z\right)+\tilde{V}^{-} \exp \left(j \sqrt{Z Y} k_{z} z\right)$ and $\tilde{I}=\sqrt{Y / Z}\left[\tilde{V}^{+} \exp \left(-j \sqrt{Z Y} k_{z} z\right)-\tilde{V}^{-} \exp \left(j \sqrt{Z Y} k_{z} z\right)\right]$, where $\tilde{V}^{+}$and $\tilde{V}^{-}$are constants. The $\mathrm{TM}_{z}$ transmission line equations of the unbounded uniaxially anisotropic medium of interest, shown in Fig. 1a, are a relation between its $z$ and $x$ or $y$ electric field components in the form of Eqs. (1). These equations are found by substituting the permittivity and permeability tensors [5]

$$
\begin{aligned}
& \overline{\bar{\varepsilon}}=\left(\begin{array}{ccc}
\varepsilon & 0 & 0 \\
0 & \varepsilon & 0 \\
0 & 0 & \varepsilon_{z}
\end{array}\right), \\
& \overline{\bar{\mu}}=\left(\begin{array}{ccc}
\mu_{\rho} & 0 & 0 \\
0 & \mu_{\rho} & 0 \\
0 & 0 & \mu
\end{array}\right),
\end{aligned}
$$

into the spectral domain Maxwell's equations for the $\mathrm{TM}_{z}$ $\left(\tilde{H}_{z}=0\right)$ modes, with the substitutions $\partial / \partial x \rightarrow j k_{x}$ and $\partial / \partial y \rightarrow j k_{y}$, as follows

$$
-\frac{d \tilde{H}_{y}}{d z}=j \omega \varepsilon \tilde{E}_{x}
$$




$$
\begin{gathered}
\frac{d \tilde{H}_{x}}{d z}=j \omega \varepsilon \tilde{E}_{y}, \\
j k_{x} \tilde{H}_{y}-j k_{y} \tilde{H}_{x}=j \omega \varepsilon_{z} \tilde{E}_{z} \\
j k_{y} \tilde{E}_{z}-\frac{d \tilde{E}_{y}}{d z}=-j \omega \mu_{\rho} \tilde{H}_{x} \\
\frac{d \tilde{E}_{x}}{d z}-j k_{x} \tilde{E}_{z}=-j \omega \mu_{\rho} \tilde{H}_{y} \\
j k_{x} \tilde{E}_{y}-j k_{y} \tilde{E}_{x}=0 .
\end{gathered}
$$

To compute the transmission line equations, first derive

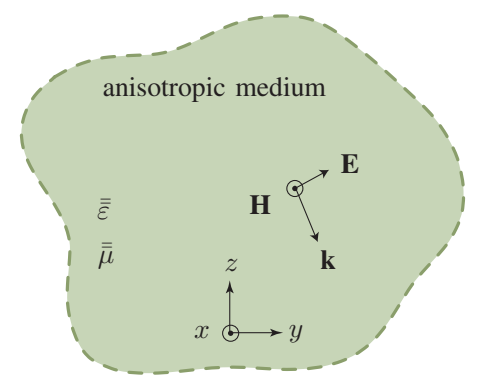

(a)

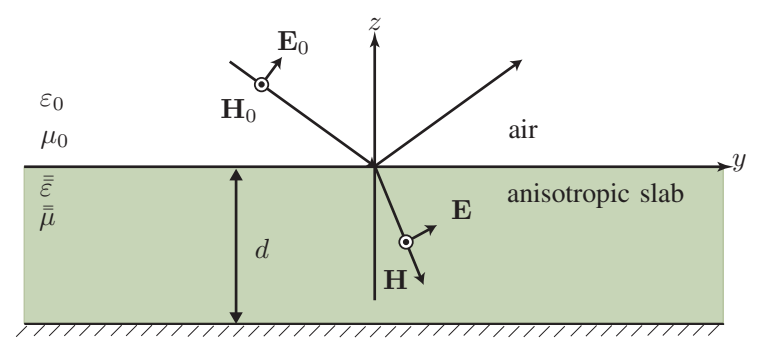

(b)

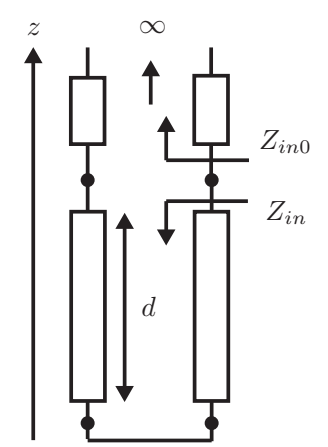

(c)

Fig. 1: Uniaxially anisotropic meta-substrate. (a) Unbounded medium. (b) Grounded slab. (c) Transmission line model.

Eq. (3c) with respect to $z$, then substitute Eqs. (3a) and (3b) into the resulting equation and further eliminate $\tilde{E}_{y}$ by using Eq.(3f) which leads to $-j k_{\rho}{ }^{2} / k_{x} \tilde{E}_{x}=\left(\varepsilon_{z} / \varepsilon\right) d \tilde{E}_{z} / d z$, where $k_{\rho}{ }^{2}=k_{x}{ }^{2}+k_{y}{ }^{2}$ is the square of the transverse wave number. This equation can be modified to the first transmission line equation, in the form of Eq. (1a), as follows

$$
\frac{\partial}{\partial z}\left[-\frac{\omega \varepsilon_{z}}{k_{x}} \tilde{E}_{z}\right]=-j\left(\frac{\omega \varepsilon}{k_{z}}\right) k_{z}\left[-\frac{k_{\rho}{ }^{2}}{k_{x}{ }^{2}} \tilde{E}_{x}\right] .
$$

To obtain the second transmission line equation, substitute $\tilde{H}_{y}$ computed from Eq. (3c) into Eq. (3e) and then substitute $\tilde{H}_{x}$ from Eq. (3d) into the resulting equation. Further, eliminating $\tilde{E}_{y}$ from Eq. (3f) in the resulting equation and defining the square of the wave number as $k^{2}=k_{\rho}{ }^{2}+k_{z}{ }^{2}=\omega^{2} \mu_{\rho} \varepsilon_{z}$ leads to $\left[k_{\rho}{ }^{2} / k_{x}{ }^{2}\right] \partial / \partial z \tilde{E}_{x}=j k_{x}\left[-k_{z}{ }^{2} / k_{x}{ }^{2}\right] \tilde{E}_{z}$. This equation can be rewritten as the second transmission line equation, in the form of Eq. (1b), as follows

$$
\frac{\partial}{\partial z}\left[-\frac{k_{\rho}{ }^{2}}{k_{x}{ }^{2}} \tilde{E}_{x}\right]=-j\left(\frac{k_{z}}{\omega \varepsilon_{z}}\right) k_{z}\left[\frac{-\omega \varepsilon_{z}}{k_{x}} \tilde{E}_{z}\right] .
$$

From the transmission line equations (4) and (5) of the uniaxially anisotropic medium, the components of the transmission line model are found by identification with Eqs. (1) as $\tilde{V}=$ $-k_{\rho}{ }^{2} / k_{x}{ }^{2} \tilde{E}_{x}, \tilde{I}=-\omega \varepsilon_{z} / k_{x} \tilde{E}_{z}, Z=k_{z} / \omega \varepsilon_{z}, Y=\omega \varepsilon / k_{z}$. As a result, $Z_{c}=k_{z} / \omega \varepsilon \sqrt{\varepsilon / \varepsilon_{z}}$ and $\beta=\sqrt{\varepsilon / \varepsilon_{z}} k_{z}$.

\section{DisPERSiON RELATION OF A UNIAXIALLY Anisotropic META-SUbSTRATE}

Figs. $1 \mathrm{~b}$ and $1 \mathrm{c}$ show the grounded meta-substrate and its $\mathrm{TM}_{z}$ transmission line model, respectively. The dispersion relation of the structure can be obtained by applying the transverse resonance condition $Z_{i n 0}+Z_{i n}=0$ [8], where $Z_{i n 0}=Z_{c 0}$ and $Z_{i n}=j Z_{c} \tan (\beta d)$ are the impedances of the semi-infinite free-space and the input impedance of the slab from its interface with the air, respectively, and $Z_{c 0}=k_{z 0} / \omega \varepsilon_{0}$ is the characteristic impedance of the free space, where $k_{z 0}$ is the wave number of the free-space along the $z$ axis. Remembering that in the slab $k_{z}= \pm \sqrt{\omega^{2} \mu_{\rho} \varepsilon_{z}-k_{\rho}^{2}}$ and in the air $k_{z 0}= \pm \sqrt{\omega^{2} \mu_{0} \varepsilon_{0}-k_{\rho}^{2}}$, the $\mathrm{TM}_{z}$ dispersion relation is obtained as

$$
\begin{aligned}
& j \frac{\sqrt{\omega^{2} \mu_{\rho} \varepsilon_{z}-k_{\rho}^{2}}}{\omega \varepsilon} \sqrt{\frac{\varepsilon}{\varepsilon_{z}}} \tan \left(\sqrt{\frac{\varepsilon}{\varepsilon_{z}}} \sqrt{\omega^{2} \mu_{\rho} \varepsilon_{z}-k_{\rho}^{2}} d\right) \pm \\
& \frac{\sqrt{\omega^{2} \mu_{0} \varepsilon_{0}-k_{\rho}^{2}}}{\omega \varepsilon_{0}}=0 .
\end{aligned}
$$

In the left-handed band of the anisotropic meta-substrate [5], $\varepsilon_{z}<0$ and $\mu_{\rho}<0$ while $\varepsilon>0$ and $\mu>0$. Then, Eq. (6) may be conveniently written using absolute values as

$$
\begin{aligned}
& \frac{\sqrt{\omega^{2}\left|\mu_{\rho}\right|\left|\varepsilon_{z}\right|-k_{\rho}^{2}}}{\omega \varepsilon} \sqrt{\frac{\varepsilon}{\left|\varepsilon_{z}\right|}} \tanh \left(\sqrt{\frac{\varepsilon}{\left|\varepsilon_{z}\right|}} \sqrt{\omega^{2}\left|\mu_{\rho}\right|\left|\varepsilon_{z}\right|-k_{\rho}^{2}} d\right) \\
& \pm j \frac{\sqrt{\omega^{2} \mu_{0} \varepsilon_{0}-k_{\rho}^{2}}}{\omega \varepsilon_{0}}=0
\end{aligned}
$$

In the particular case of the corresponding isotropic lefthanded grounded slab with $\varepsilon_{z}=\varepsilon<0$ and $\mu_{\rho}=\mu<0$, 
TABLE I: Characteristics of the $\mathrm{TM}_{z}$ modes of a uniaxially anisotropic meta-substrate (Fig. 4).

\begin{tabular}{c|ccccc} 
Modes & $-\operatorname{Re}\left(k_{\rho} / k_{0}\right)$ & $-\operatorname{Im}\left(k_{\rho} / k_{0}\right)$ & $\operatorname{Re}\left(k_{z 0} / k_{0}\right)$ & $\operatorname{Im}\left(k_{z 0} / k_{0}\right)$ & Characteristic \\
\hline $\mathrm{A}$ & $1<$ and $<\sqrt{\varepsilon_{z} \mu_{\rho}}$ & $=0$ & $=0$ & $<0$ & surface mode \\
$\mathrm{B}$ & $1<$ and $<\sqrt{\varepsilon_{z} \mu_{\rho}}$ & $=0$ & $=0$ & $>0$ & non-physical \\
$\mathrm{C}$ & $<1$ & $>0$ & $>0$ & $>0$ & leaky mode
\end{tabular}

Eq. (6) is reduced to [9]

$$
\begin{aligned}
& \frac{\sqrt{\omega^{2}|\mu||\varepsilon|-k_{\rho}{ }^{2}} \tan \left(\sqrt{\omega^{2}|\mu||\varepsilon|-k_{\rho}{ }^{2}} d\right)}{\omega|\varepsilon|} \\
& \pm j \frac{\sqrt{\omega^{2} \mu_{0} \varepsilon_{0}-k_{\rho}{ }^{2}}}{\omega \varepsilon_{0}}=0 .
\end{aligned}
$$

\section{DISPERSION ANALYSIS}

\section{A. Drude/Lorentz Permittivity/Permeability Dispersion Mod-} els

The anisotropic meta-substrate of interest is inherently dispersive [5]. The wires along the $z$ axis exhibit an effective Drude permittivity along this axis, $\varepsilon_{z} / \varepsilon_{0}=\varepsilon_{r \infty}-\omega_{e p}{ }^{2} / \omega^{2}$ [10], and the loops between adjacent mushrooms exhibit an effective Lorentz permeability in the $\rho$ plane, $\mu_{\rho} / \mu_{0}=1-$ $F \omega^{2} /\left(\omega^{2}-\omega_{m 0}{ }^{2}\right)[11]$. The permittivity and permeability of the structure along the other directions are the same as the host medium. Fig. 2 shows typical dispersion curves of the permittivity and permeability.

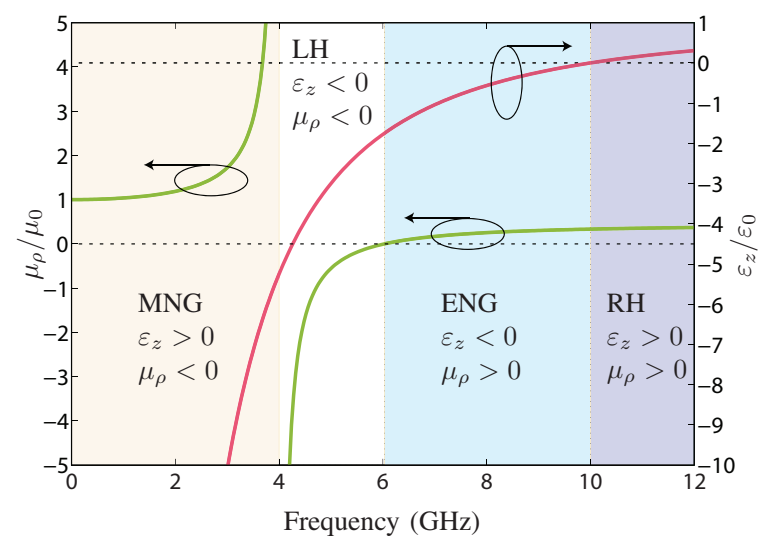

Fig. 2: Dispersion response of the permittivity $\varepsilon_{z} / \varepsilon_{0}$ (Drude model) and permeability $\mu_{\rho} / \mu_{0}$ (Lorentz model) for the parameters $\varepsilon_{r \infty}=1, F=0.56, \omega_{m 0}=2 \pi \times 4 \times 10^{9}$, $\omega_{e p}=2 \pi \times 10 \times 10^{9}$ and $d=60 \mathrm{~mm}$ [9]. The following regions may be distinguished: negative permittivity or electric negative (ENG), left-handed (LH), negative permeability or magnetic negative $(\mathrm{MNG})$ and right-handed $(\mathrm{RH})$.

\section{B. Limit Case of an Isotropic Grounded Slab}

The time dependency $\exp (+j \omega t)$ is assumed, corresponding to the outgoing-wave space dependency $\exp \left(-j \mathbf{k}_{0} \cdot \mathbf{r}\right)=\exp \left(-j \mathbf{k}_{\rho} \cdot \boldsymbol{\rho}\right) \exp \left(-j \mathbf{k}_{z 0} \cdot \mathbf{z}\right)=\exp \left(-j k_{\rho} \rho\right)$ $\exp \left(-j k_{z 0} z\right) \quad=\quad \exp \left(-j \operatorname{Re}\left(k_{\rho}\right) \rho\right) \quad \exp \left(\operatorname{Im}\left(k_{\rho}\right) \rho\right)$ $\exp \left(-j \operatorname{Re}\left(k_{z 0}\right) z\right) \exp \left(\operatorname{Im}\left(k_{z 0}\right) z\right)$. In the considered lefthanded region of Fig. 2, $k_{\rho}$ is negative (propagation along $-\boldsymbol{\rho}$ ), and we consider $-\operatorname{Re}\left(k_{\rho}\right)$ and $-\operatorname{Im}\left(k_{\rho}\right)$ in the study. First, the dispersion curves of an isotropic grounded slab, which is the particular case of the anisotropic meta-substrate with $\varepsilon=\varepsilon_{z}$ and $\mu=\mu_{\rho}$, are plotted for a comparison reference. Next, the dispersion behavior of a slightly anisotropic grounded slab, exhibiting $\varepsilon=2 \varepsilon_{z}$ and $\mu=2 \mu_{\rho}$, is compared with the isotropic grounded slab. The corresponding dispersion curves are shown in Fig. 3. This graph shows that the introduction of anisotropy progressively shifts the $\mathrm{TM}_{2}$ mode toward higher frequencies, while the $\mathrm{TM}_{1}$ is essentially unchanged. The indicated evanescent mode decays both in the slab and in the air along the $z$ axis. As expected, extension of the modes in the ENG region $(f>6.03 \mathrm{GHz}$ ) exhibit very high loss $\left(-\operatorname{Im}\left(k_{\rho} / k_{0}\right)\right)$ and therefore vanish quickly along the $\rho$ axis. Moreover, the mode index $m$ [Eq. (8)] increases with decreasing frequency, which is a trend opposite to that of conventional right-handed dielectric slabs.

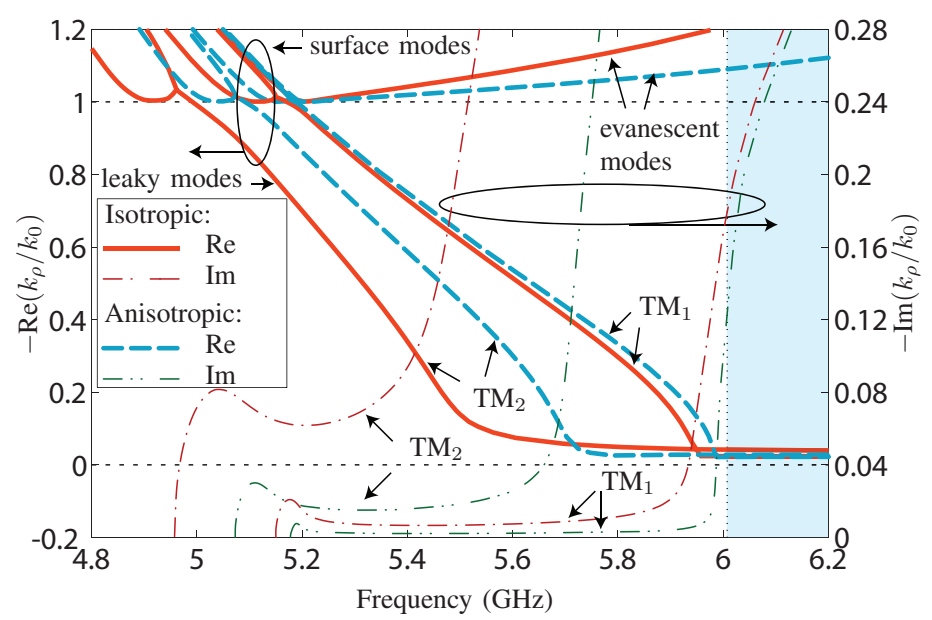

Fig. 3: Comparing the dispersion characteristics of an isotropic slab with the parameters of Fig. 2 while $\varepsilon=\varepsilon_{z}$ and $\mu=\mu_{\rho}$ with the thickness $d=60 \mathrm{~mm}$, with a slightly anisotropic slab with the same parameters as Fig. 2 and $\varepsilon=2 \varepsilon_{z}$ and $\mu=2 \mu_{\rho}$. The isotropic curves are in agreement with the results reported in [9].

\section{Uniaxially Anisotropic Meta-substrate}

The $\mathrm{TM}_{z}$ modes of a uniaxially anisotropic meta-substrate with the parameters of Fig. 2 and $\varepsilon=1.4 \varepsilon_{0}$ and $\mu=\mu_{0}$ are shown in Figs. 4. Three modes, labeled $A, B$ and $C$ and summarized in Tab. I, are visible. They correspond to a surface mode $(A)$ with $1<-\operatorname{Re}\left(k_{\rho} / k_{0}\right)<\sqrt{\left|\varepsilon_{z}\right|\left|\mu_{\rho}\right|}$, $-\operatorname{Im}\left(k_{\rho} / k_{0}\right)=0, \operatorname{Re}\left(k_{z 0} / k_{0}\right)=0$ and $\operatorname{Im}\left(k_{z 0} / k_{0}\right)<0$, a 


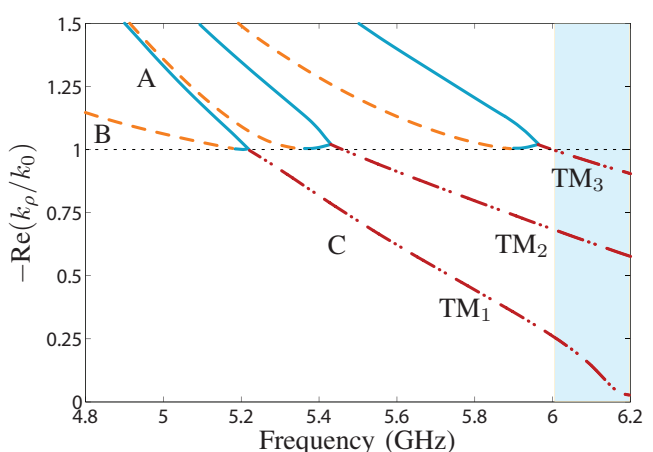

(a)

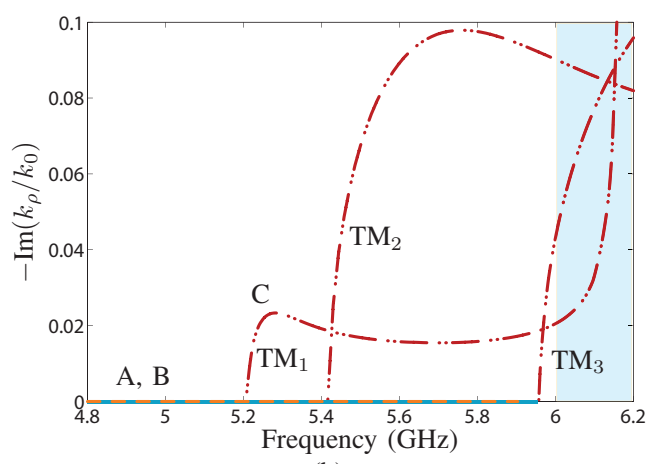

(b)

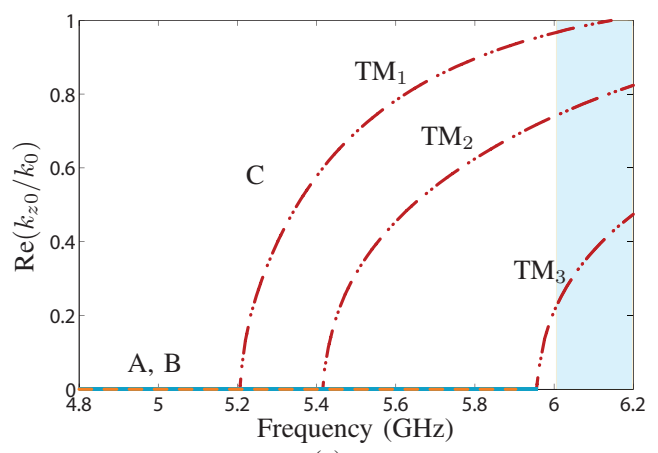

(c)

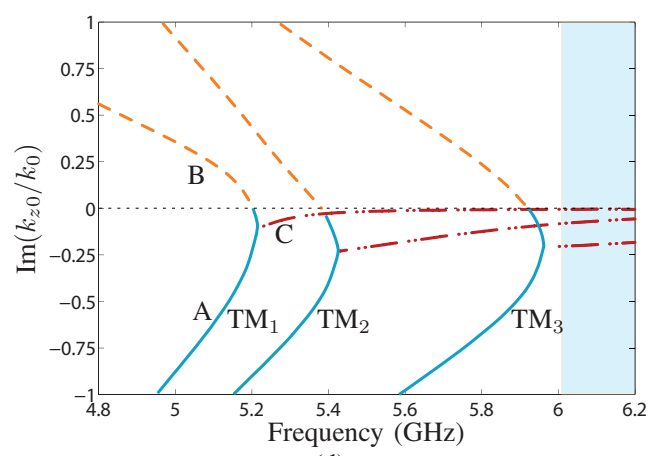

(d)

Fig. 4: $\mathrm{TM}_{z}$ dispersion curves for a uniaxially anisotropic meta-substrate with the parameters of Fig. 2 and $\varepsilon=1.4 \varepsilon_{0}$ and $\mu=\mu_{0}$ and the thickness $d=60 \mathrm{~mm}$. (a) $\operatorname{Re}\left(k_{\rho} / k_{0}\right)$. (b) $\operatorname{Im}\left(k_{\rho} / k_{0}\right)$. (c) $\operatorname{Re}\left(k_{z 0} / k_{0}\right)$. (d) $\operatorname{Im}\left(k_{z 0} / k_{0}\right)$.

non-physical mode $(B)$ with $1<-\operatorname{Re}\left(k_{\rho} / k_{0}\right)<\sqrt{\left|\varepsilon_{z}\right|\left|\mu_{\rho}\right|}$, $-\operatorname{Im}\left(k_{\rho} / k_{0}\right)=0, \operatorname{Re}\left(k_{z 0} / k_{0}\right)=0$ and $\operatorname{Im}\left(k_{z 0} / k_{0}\right)>0$, and a leaky mode $(C)$ with $-\operatorname{Re}\left(k_{\rho} / k_{0}\right)<1,-\operatorname{Im}\left(k_{\rho} / k_{0}\right)>0$, $\operatorname{Re}\left(k_{z 0} / k_{0}\right)>0$ and $\operatorname{Im}\left(k_{z 0} / k_{0}\right)>0$. Comparison between Figs. 3 and 4 show that, in the anisotropic slab, as the order $m$ of the mode increases, the surface-leaky transition frequency also increases, in contrast to the case of the isotropic structure with low isotropy, which means that high anisotropy inverts the frequency order of the modes. Moreover, the leaky-modes in the anisotropic meta-substrate exhibit less radiation loss as compared to the isotropic grounded slab, leading to higher directivity in leaky-wave antennas. Finally, the anisotropic meta-substrate leaky-modes exhibit a smooth transition with lower loss into the ENG band.

\section{CONCLuSion}

The $\mathrm{TM}_{z}$ spectral transmission line model of a uniaxially anisotropic mushroom-type grounded meta-substrate has been presented. The corresponding dispersion relation was derived by applying the transverse resonance condition. A particular cases of an isotropic grounded-slab and a slightly anisotropic grounded slab were compared with the anisotropic metasubstrate in their left-handed frequency band. The analysis of the meta-substrate dispersion has shown that the leakymodes exhibit a leakage factor which may be conveniently designed to very low values for super highly directive leakywave antennas.

\section{REFERENCES}

[1] P. M. T. Ikonen, K. N. Rozanov, A. V. Osipov, P. Alitalo, and S. A. Tretyakov, "Magnetodielectric substrates in antenna miniaturization: potentials and limitations," IEEE Trans. Antennas Propagat., vol. 54, no. 11, pp. 3391-3399, 2006.

[2] H. Mosallaei and K. Sarabandi, "Design and modeling of patch antenna printed on magneto-dielectric embedded-circuit metasubstrate," IEEE Trans. Antennas Propagat., vol. 55, no. 1, pp. 45-52, Jan. 2007.

[3] M. Coulombe, H. V. Nguyen, and C. Caloz, "Substrate integrated artificial dielectric (SIAD) structure for miniaturized microstrip circuits," IEEE Antennas and Wireless Propagat. Lett., vol. 6, no. 6, pp. 575-579, Dec. 2007.

[4] C. Caloz and T. Itoh, Electromagnetic Metamaterials: Transmission line Theory and Microwave Applications, Wiley-IEEE Press, NJ, 2006.

[5] H. V. Nguyen and C. Caloz, "Anisotropic backward-wave metasusbtrate and its application to a microstrip leaky-wave antenna," in Proc. CNC/USNC URSI National Radio Science Meeting, Ottawa, ON, Canada, July 2007.

[6] D. Sievenpiper, L. Zhang, R. F. J. Broas, N. G. Alexopolous, and E. Yablonovitch, "High-impedance electromagnetic surfaces with a forbidden frequency band," IEEE Trans. Microwave Theory Tech., vol. 47, no. 11, pp. 2059-2074, Nov. 1999.

[7] L. B. Felsen and N. Marcuvitz, Radiation and Scattering of Waves, Prentice-Hall/IEEE Press, NY, 1996.

[8] N. Marcuvitz (Ed.), Waveguide Handbook, chapter 8, McGraw-Hill, NY, 1951.

[9] P. Baccarelli, P. Burghignoli, A. Galli, P. Lampariello, G. Lovat, S. Paulotto and G. Valerio, chap. 13 in F. Capolino (editor), Theory and Phenomena of Metameterials, , CRC Press, FL, 2009.

[10] S. A. Tretyakov, Analytical Modeling in Applied Electromagnetics, Artech House, Norwood, MA, 2003.

[11] J. B. Pendry, A. J. Holden, D. J. Robbins, and W. J. Stewart, "Magnetism from conductors and enhanced non-linear phenomena," IEEE Trans. Microwave Theory Tech., vol. 47, no. 11, pp. 2075-2084, Nov. 1999. 